LAPS and the ALPPS was $85.7 \%(6 / 7)$ and $78.6 \%(11 / 14)$. The incidence of major complications was $36.4 \%(4 / 11)$ of the ALPPS group and $50.0 \%(3 / 6)$ of the LAPS group after the 2 stages operation. One patient died of the ALPPS group. Additionally, the median increase in FLR, median operative time and blood loss during the two stages of the LAPS were similar to those subjected to ALPPS.

Conclusions LAPS has a potential advantage in eliminating major complications of PHLF associated with classic ALPPS. LAPS may achieve the same effect of promoting significant growth of the FLR in patients with HBV-related HCC, albeit at the cost of longer interval time.

\section{IDDF2020-ABS-0069 PREDICTION OF MICROVASCULAR INVASION BEFORE SURGERY IN PATIENTS WITH HEPATOCELLULAR CARCINOMA: A NOMOGRAM MODEL BASED ON INFLAMMATORY MARKERS}

${ }^{1}$ Qianwen Zeng*, ${ }^{2}$ Han Xiao, ${ }^{3}$ Tingfan Wu, ${ }^{3}$ Xin Li, ${ }^{4}$ Sui Peng, ${ }^{5}$ Ming Kuang. 'Zhongshan School of Medicine, Sun Yat-sen University, China; ${ }^{2}$ Division of Interventional Ultrasound, The First Affiliated Hospital of Sun Yat-sen University, China; ${ }^{3}$ GE Healthcare, Beijing, China; ${ }^{4}$ Clinical Trials Unit, Department of Gastroenterology, The First Affiliated Hospital of Sun Yat-sen University, China; ${ }^{5}$ Department of Liver Surgery, Cancer Center, Precision Medicine Institute, The First Affiliated Hospital of Sun Yat-sen University, China

\subsection{6/gutjpl-2020-IDDF.149}

Background Microvascular invasion (MVI) remains a risk factor for tumor recurrence and metastasis in hepatocellular carcinoma (HCC). No effective and well-recognized method can detect MVI before surgery. Inflammatory markers reflect the immune environment and have been proven to be related to prognosis as well as the presence of MVI in HCC. We aimed to establish an MVI predictive model based on inflammatory markers.

Methods Data of 1058 cases of HCC patients treated in the First Affiliated Hospital of Sun Yat-sen University from November 2003 to December 2015 were collected. In a ratio of $7: 3$, patients were divided into the training group (740 cases) and the validating group (318 cases). Inflammatory factors related to MVI diagnosis in HCC patients were selected by LASSO regression analysis, and were then integrated into an 'Inflammatory Score'. A prognostic Nomogram model was established by combining the Inflammatory Score and the independent factors determined by multivariate logistic regression analysis. The consistency index (C-index) and the area under the curve (AUC) were used to evaluate the predictive efficacy of the model.

Results A total of 1058 HCC patients were included in this retrospective study, 430 of whom $(40.6 \%)$ were diagnosed with MVI. Sixteen inflammatory factors, including neutrophil, neutrophil to lymphocyte ratio, platelet to lymphocyte ratio, etc., were selected by LASSO regression analysis to establish an Inflammatory Score. Multivariate logistic regression analysis showed that Inflammatory Score $(\mathrm{OR}=2.186,97.5 \% \mathrm{CI}$ : 1.656-2.950), age (OR $=0.987,97.5 \% \mathrm{CI}: 0.973-1.000)$, alpha fetoprotein $(\mathrm{OR}=1.923,97.5 \%$ CI: 1.380-2.690), tumor size $(\mathrm{OR}=2.308,97.5 \% \mathrm{CI}$ : 1.656-3.220) were independent factors in the diagnosis of MVI in HCC patients. These four factors were then used to establish a Nomogram for MVI prediction. The C-index of the Nomogram prediction model was 0.72 . The AUC for the training and validating group were 0.720 and 0.721 , respectively.
Conclusions The Nomogram prediction model drawn in this study has a high prognostic value, which is capable of improving the diagnosis efficiency of MVI in HCC patients.

\section{IDDF2020-ABS-0078 IMMUNOSCORE CLASSIFICATION FROM HEPATOCELLULAR CARCINOMA HISTOPATHOLOGY IMAGES USING DEEP LEARNING: A PRELIMINARY STUDY}

${ }^{1}$ Qiaofeng Chen*, ${ }^{2}$ Yunquan Gu, ${ }^{2}$ Ruixuan Wang, ${ }^{3}$ Han Xiao, ${ }^{4}$ Sui Peng, ${ }^{5}$ Ming Kuang. ${ }^{1}$ Department of Gastroenterology, the First Affiliated Hospital, Sun Yat-sen University, China; ${ }^{2}$ School of Data and Computer Science, Sun Yat-sen University, China; ${ }^{3}$ Division of Interventional Ultrasound, the First Affiliated Hospital, Sun Yat-sen University, China; ${ }^{4}$ Clinical Trials Unit, the First Affiliated Hospital, Sun Yat-sen University, China; ${ }^{5}$ Department of Liver Surgery, the First Affiliated Hospital, Sun Yat-sen University, China

\subsection{6/gutjnl-2020-IDDF.150}

Background Immunotherapy is a recent advance for the treatment of hepatocellular carcinoma (HCC). Immunoscore assessment plays a critical role in precision immunotherapy and can predict prognosis in patients with HCC. This study aims to develop a deep-learning model to automated analyze histopathology images for classification of immunoscore (CD3 or CD8, 0-2 vs. 3-4) in HCC.

Methods We trained a patch-based deep convolutional neural network (Resnet-18) on whole-slide images to automatically classify immunoscore into $0-2$ or 3-4. The data were randomly split into a training and testing dataset. The performance was first estimated on the training dataset with ninefolded cross-validation and then further validated on the testing dataset. Cross-entropy was used as a model-optimized loss function and the accuracy as well as the area under the receiver operating characteristic curve (AUC) were calculated for the identification values. Heatmaps were also generated by our model to visualize the regions the most associated with the classification.

Results We included 28 images from a study cohort of 28 HCC patients for training (18 images) and testing (10 images) the model. After iterative training, an optimized architecture

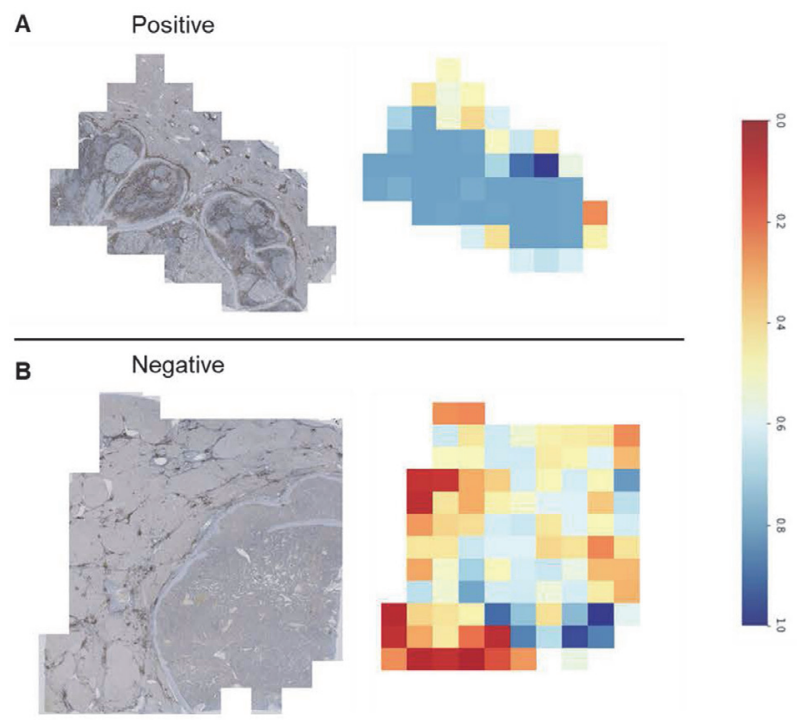

Abstract IDDF2020-ABS-0078 Figure 1 Heatmaps of patch-based tissue slides generated by the model in the testing dataset 
achieved an AUC of 0.71 was used as our final model. For validation on the testing dataset, the model yielded an accuracy of $90 \%$ and AUC of 0.93 (95\% CI: 0.76 to 1.00 ) while the percentage of patches positively classified, and outperforms average of the probabilities of the corresponding patches (accuracy 70\%; AUC 0.79, 95\% CI: 0.50 to 1.00) using the same optimal threshold of 0.33 . The heatmaps show that almost all of patches are highly identified to show the regions of immunoscore ((figure 1) A. Immunoscore of 3-4 [positive]. B. Immunoscore of $0-2$ [negative]).

Conclusions The automated deep-learning model achieved good performance and could potentially assist clinicians in the identification of HCC patients who are more likely to respond to immunotherapy, or at least, providing second opinions on therapeutic decision-making.

\section{IDDF2020-ABS-0088 COMPARING NON-INVASIVE TESTS FOR PREDICTION OF FIBROSIS IN NON- ALCOHOLIC FATTY LIVER DISEASE}

Wilson Siu*, Yusuke Onishi, Ashis Mukhopadhya. Department of Digestive Disorders, Aberdeen Royal Infirmary, UK

\subsection{6/gutjnl-2020-IDDF.151}

Background Non-alcoholic fatty liver disease (NAFLD) is becoming the most common type of chronic liver disease with an estimated worldwide prevalence of $25 \%$. It is a spectrum of disease that ranges from simple steatosis to advanced fibrosis. Non-invasive tests play an important role in identifying patients with fibrosis that require further investigation and follow up. The aim of the study is to evaluate the diagnostic accuracy of different non-invasive scoring tests.

Methods Patients with NAFLD who underwent liver stiffness measurement (LSM) by FibroScan at Aberdeen Royal Infirmary between 2013 and 2016 were retrospectively included in our study. Patients' demographic, clinical and laboratory data were collected closest from the date of the FibroScan. NAFLD fibrosis score (NFS), APRI, FIB-4 and BARD scores were calculated. For this study, clinically significant fibrosis (CSF) is defined as LSM $>7 \mathrm{kPa}$ and advanced fibrosis is defined as LSM $>12 \mathrm{kPa}$. The diagnostic accuracy of the four fibrosis scores was examined by calculating the area under the receiver operating characteristic curve (AUROC). The sensitivity, specificity, positive predictive value and negative value were calculated using optimal cut-offs calculated by Youden index.

Results Of the 863 patients included in this study, 498 $(57.7 \%)$ were male and the mean age was 54.4 years $(\mathrm{SD}=14.7)$. The mean $\mathrm{BMI}$ was $32.6 \quad(\mathrm{SD}=6.4) .48 \%$ of patients had CSF with LSM $>7 \mathrm{kPa}$ and $28 \%$ had advanced fibrosis with LSM $>12 \mathrm{kPa}$. For CSF, AUROC curve values were: NFS 0.77 (95\% CI, 0.73-0.80), FIB-4 0.74 (95\% CI, $0.71-0.78)$, APRI 0.74 (95\% CI, 0.70-0.78) and BARDS 0.65 (95\% CI, 0.65-0.74). For advanced fibrosis, the AUROC curve values were: NFS 0.83 (95\% CI, 0.80 - 0.87), FIB-4 0.79 (95\% CI, 0.75-0.83), APRI 0.75 (95\% CI, 0.71 to 0.79 ) and BARDS 0.75 (95\% CI, 0.71-0.79).

Conclusions All fibrosis scores were superior at detecting advanced fibrosis (LSM>12kPa) compared to CSF (LSM > $7 \mathrm{kPa})$. NFS showed a superior diagnostic accuracy of fibrosis compared to other scores.

\section{IDDF2020-ABS-0092 PREDICTION OF POST-HEPATECTOMY LIVER FAILURE IN PATIENTS WITH HEPATOCELLULAR CARCINOMA BASED ON GD-EOB-DTPA-ENHANCED MRI: A LF SCORING MODEL}

Yuyan Chen*. The First Affiliated Hospital of Sun Yat-sen University, China

\subsection{6/gutjnl-2020-IDDF.152}

Background The purpose of this study was to establish a preoperative clinical-radiomics prediction model of post-hepatectomy liver failure (PHLF) in patients with hepatocellular carcinoma(HCC) and to predict clinical outcome of HCC patients who suffered PHLF.

Methods The study included 555 HCC patients who underwent hepatectomy first time from January 2015 to December 2019 in The First Affiliated Hospital of Sun Yat-sen University and Sun Yat-sen University Cancer Center. Gd-EOB-DTPAEnhanced MRI was performed within 30 days before surgery. Patients in this study didn't have other tumors or serious organic diseases and were followed up after liver resections for 90 days. We obtained 60720 MR images from 555 patients (an ROI is shown as figure 1), including 390 cases as training data and 165 cases as independent testing data with the standard of PHLF as ISGLS. Through the multiple-model fusion algorithm, we extracted 1044 features per patient from his MR images, screening out feature sets of high contribution by RFE-SVM algorithm and transforming them to FF scores. Clinical indicators, radiologic features and FF scores were included in our LF scoring model through LDA(Linear discriminant Analysis) algorithms.

Results The AUC of LF scoring model reached 0.953 (95\%CI $0.953-0.963)$ and 0.945 (95\%CI $0.941-0.980)$ in the training

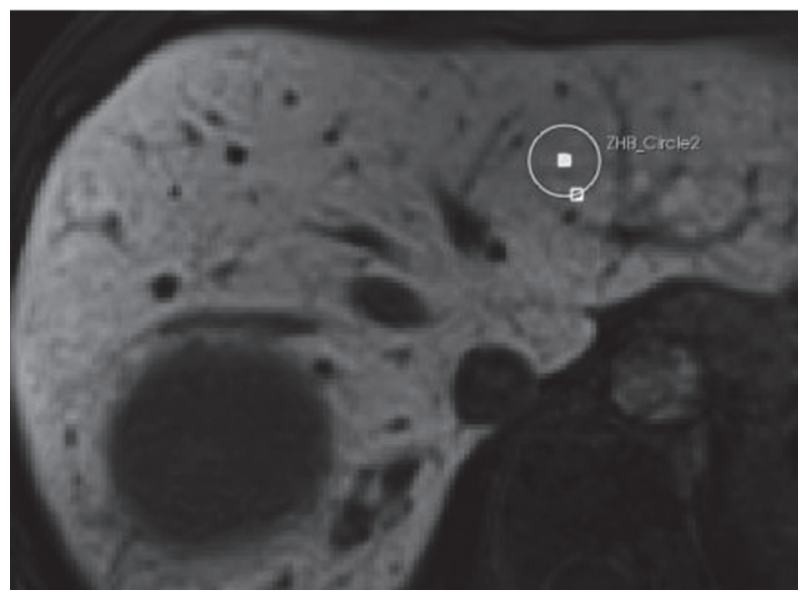

Abstract IDDF2020-ABS-0092 Figure 1 ROI of a certain patient

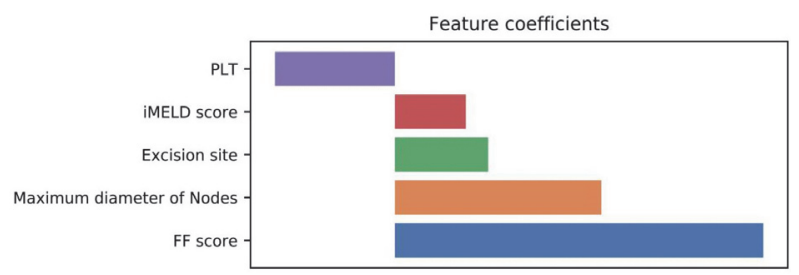

Abstract IDDF2020-ABS-0092 Figure 2 Feature coefficients in the clinical-radiomics model 